\title{
Primitive neuroectodermal tumor: Report of two cases and review of the literature
}

\author{
R. Hage, MD, ${ }^{a}$ V. A. M. Duurkens, MD, ${ }^{a}$ C. A. Seldenrijk, MD, $P h D,{ }^{b}$ A. Brutel de la Rivière, $M D, P h D,{ }^{c}$ \\ H. A. van Swieten, MD, PhD, ${ }^{c}$ and J. M. M. van den Bosch, MD, PhD, ${ }^{a}$ Nieuwegein, The Netherlands
}

$\mathrm{T}$ he malignant small round-cell tumors of the thoracopulmonary region were first described by Askin in 1979. ${ }^{1}$ These tumors are characterized by neuroectodermal derivation and anatomic distribution. Currently, Ewing sarcoma (ES), primitive neuroectodermal tumors (PNETs), and malignant small round-cell tumors of the thoracopulmonary region are suggested to be different manifestations of a single tumor family. However, these tumors are rare and often occur in childhood or adolescence. Only a few series have been described in the past. We review 2 cases of patients who were referred to our clinic, followed by a review of literature.

\section{Clinical Summaries}

PATIENT 1. A 13-year-old boy presented with a right-sided palpable painful mass on his chest for 1 month and thoracic pain for 5 months.

There was no pain elsewhere, nor was there any dyspnea or constitutional symptoms, such as fever and weight loss. He had no smoking history. At physical examination, there was a slightly prominent lateral chest wall on the right, without other signs of pathology. A chest x-ray film showed an abnormal consolidation in the right lateral thorax on the level of the eighth dorsal rib and discrete pleural effusion in the right hemithorax (Figure 1).

Thoracoscopy showed a normal right upper lobe and an adhesion of the middle lobe to the anterolateral chest wall with thickening of the visceral pleura. The parietal pleura under this adhesion was irregular and red colored. There was some hemorrhagic fluid in the right thorax, showing no clear malignancy on posteroanterior examination. Bone scintigraphy results were positive for the right seventh rib from the anterolateral to the dorsal position, without other hot spots. Thoracotomy was performed for incisional biopsy of the tumor.

Histologically, the tumor had a solid predominant lobular growth pattern (Figure 2, A). There were fibrovascular septa between the tumor cells. There were large areas of necrosis in patient

From the Heart Lung Centre Utrecht (HLCU), St Antonius Hospital, Departments of Pulmonology, ${ }^{\mathrm{a}}$ Pathology, ${ }^{\mathrm{b}}$ and Thoracic Surgery, ${ }^{\mathrm{c}}$ Nieuwegein, The Netherlands.

Received for publication Jan 4, 2002; accepted for publication March 4, 2002

Address for reprints: J. M. M. van den Bosch, MD, St Antonius Hospital, Department of Pulmonology, PO Box 2500, Niuewegein, The Netherlands 3430 EM (E-mail: j.vandenbosch@antonius.net).

J Thorac Cardiovasc Surg 2002;124:833-6

Copyright (C) 2002 by The American Association for Thoracic Surgery

$0022-5223 / 2002 \$ 35.00+0 \quad \mathbf{1 2 / 5 4 / 1 2 4 2 6 2}$

doi: $10.1067 / \mathrm{mtc} .2002 .124262$
1 , whereas necrosis was absent in patient 2 . The cells were round and had scant cytoplasm with inconspicious nucleoli. Mitotic figures were numerous. Rosettes were only focally identified. Immunohistochemistry revealed tumor cells strongly positive for monoclonal antibody-1, Leu-7, neuron-specific enolase (NSE), vimentine, and CD99, a product of the MIC2 gene on the chromosome (Figure 2, B). Tumor cells were negative for MNF 116, Lu-5, neurofilament, desmine, smooth muscle actin, S100, and CD45 (common leukocyte antigen). Focally, synaptofysine and chromogranine were positive.

In both cases electron microscopy showed round-to-oval nuclei, massive glycogen, and dense core neurosecretory granules with a diameter of 160 to $200 \mathrm{~nm}$. Because of these histologic, immunohistochemical, and electron microscopic characteristics, the tumor was diagnosed as a PNET.

With the diagnosis of PNET, the patient underwent subsequent thoracic surgery. There was resection of the large chest wall soft-tissue mass of $11 \times 7 \times 4.8 \mathrm{~cm}$, as well as the affected ribs (Figure 3). The right fifth, sixth, and seventh ribs were resected, as was the ventral part of the serratus anterior muscle and the intercostal bundle of the fourth and seventh intercostal space. The chest wall defect was closed with Marlex mesh, as well as reconstruction with the latissimus dorsi muscle and the dorsal part of the serratus anterior muscle by the plastic surgeon. There was no macroscopic direct lung invasion or lymph node involvement. Postoperatively, there were no complications. Afterward, the patient was referred to a pediatric oncology clinic, where he was treated with combination chemotherapy (cyclophosphamide, doxorubicin, dactinomycin, and vincristine). There were no signs of tumor on computed tomographic scanning of the thorax. Fourteen months after his initial diagnosis, he presented with bone metastasis in the lumbar spine (L1), for which he received local radiotherapy. Shortly thereafter, there were multiple intrapulmonary and bone metastases. Single-drug carboplatin infusions were not successful. The patient died at home, 2 years after his initial diagnosis.

PATIENT 2. A 48-year-old woman was referred to our clinic because of severe dorsal left-sided thoracic pain lasting several weeks. There was no dyspnea, fatigue, or weight loss. Over 32 years, she had been smoking 1 or 2 cigarettes a day. Physical examination was unrewarding. On the chest x-ray film, there was an oval tumor with sharp borders in the left dorsal thorax. Fluoroscopy showed that the tumor was attached to the chest wall. This was confirmed by means of computed tomographic scanning of the thorax. Fiberoptic bronchoscopy showed a completely normal airways system.

Bone scintigraphy showed increased uptake in the eighth dorsal left rib, without other hot spots. Ultrasound examination of the upper abdomen revealed no metastasis. 


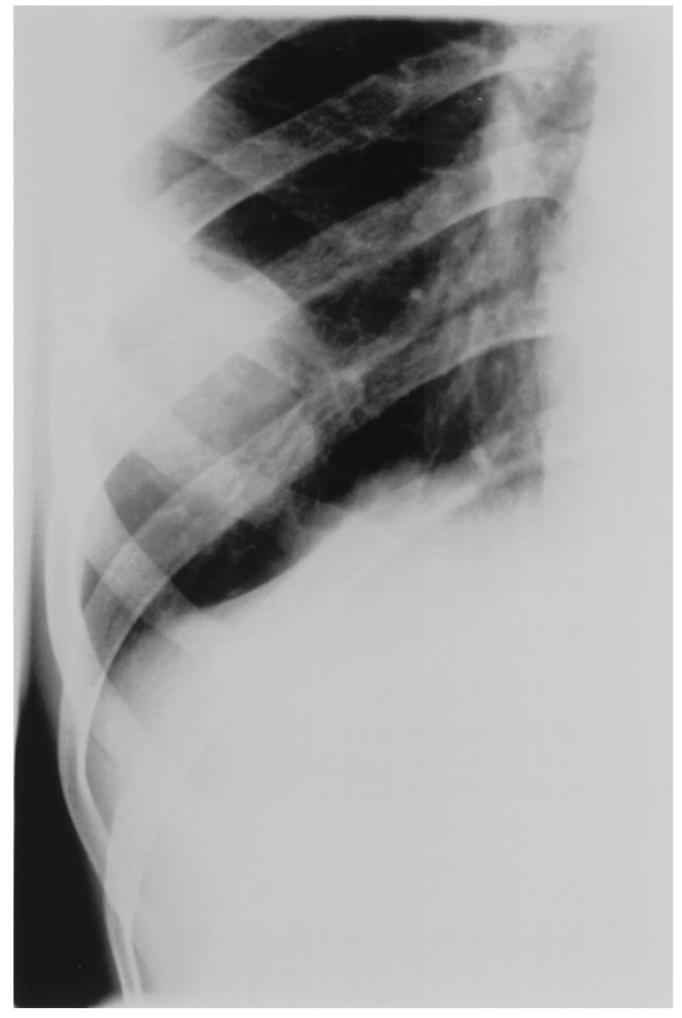

Figure 1. Chest radiograph showing a large solid mass involving the right thoracic wall.

A transthoracic needle biopsy was performed twice, and although no certain PA diagnosis could be made, there was a high suspicion of malignancy.

On thoracotomy, there was normal lung tissue and a large smooth soft-tissue mass on the chest wall with a diameter of 6.5 $\mathrm{cm}$, with local destruction of the ribs. Dorsal parts of ribs 7,8 , and 9 were also removed to attempt a curative resection. The postoperative course was uneventful.

Seven months later, the patient was admitted with pain in the left thorax and in the right upper leg. Magnetic resonance imaging (MRI) of the thorax and bone scintigraphy revealed local recurrence and multiple bone metastases in the skull, lumbar spine, and right proximal femur. The patient was given 6 courses of etoposide, vincristin, actinomycin D, ifosfamide, and Adriamycin chemotherapy. Subjectively, the patient improved because the pain in her right leg diminished. Objectively, there was stable disease.

Because of the large tumor bulk in the thorax, new chemotherapy did not seem a good option. Instead, a rethoracotomy was done 16 weeks after the start of chemotherapy. Tumor was seen in the left dorsal thorax, adherent to the lung. Also, a metastasis on the diaphragm was seen. After the debulking with resection of a segment of the apex of the left lower lobe, the metastasis on the diaphragm was extirpated. Four weeks after the rethoracotomy, the patient received thoracic irradiation with a total dose of $1600 \mathrm{cGy}$ in 2 fractions in 7 days, as well as an $800-c G y$ irradiation on the right femur (one dose). This was followed by 2 courses of cyclophosphamide, thiotepa, and carboplatin chemotherapy. Eleven months after the rethoracotomy, the patient received one treatment of palliative cranial irradiation because of symptomatic brain metastasis and died several weeks later, 2 years after the initial diagnosis.

\section{Discussion}

These 2 cases illustrate 2 different presentations and the aggressiveness of the PNET. Both patients, one child and one adult, presented with a chest wall localization of a PNET, also called Askin tumor. In case 1 a 13-year-old boy presents with a tumor from the right-sided lateral chest wall. In case 2 a 48-year-old woman presents with a chest wall tumor and local rib destruction.

Since this tumor was first described by Askin and associates ${ }^{1}$ in a series of 20 patients, only a few retrospective series and sporadic case reports have been described in literature. This rare malignant tumor is mostly found in children and is even more rare in young adults, as in case 2 .

The differential diagnosis in chest wall tumors in young patients includes ES/PNET, rhabdomyosarcoma, neuroblastoma, and lymphoma. Diagnosis of these poorly differentiated, small roundcell malignancies is very difficult when only based on light microscopy.

In 1979, Askin and associates ${ }^{1}$ suggested a neuroectoderm origin on the basis of morphologic and ultrastructural findings. In 1986, Linnoila and colleagues ${ }^{2}$ were the first who confirmed a neural phenotype by using immunohistochemistry with NSE. Although the positivity for NSE could not always be found by other investigators and the value of NSE as neural marker can be debated, other well-recognized neural markers can confirm the neural phenotype. ${ }^{3}$ In our study NSE results were negative in both cases, whereas monoclonal antibody-1 results were positive.

This malignant tumor typically involves the extrapulmonary tissue of the chest wall and the paravertebral structures as soft tissue and periosteum. When this tumor originates in the chest wall, as in the above-described cases, it is also called Askin tumor. Rarely it might also involve the lung parenchyma, either as a primary pulmonary tumor or after local spread of an adjacent tumor. ES and PNET are histologically similar and share a specific chromosomal abnormality, most commonly the reciprocal $\mathrm{t}(11,22)(\mathrm{q} 24, \mathrm{q} 12)$ translocation. They are therefore at least closely related and probably different manifestations of one tumor type, with only anatomically different locations.

In our study both patients present with chest wall pain, the most common symptom of a PNET (50\%). ${ }^{4}$ The other most common symptoms are chest wall mass (47\%), dyspnea (20\%), and fever $(17 \%)$. When located in the thoracopulmonary region, the tumor site involves a chest wall mass $(40 \%)$, rib involvement $(37 \%)$, or the lung parenchyma alone or with chest wall involvement $(23 \%)$.

Computed tomography (CT) and MRI of the thorax mostly show large tumors, often with a heterogeneous appearance because of areas of hemorrhage and necrosis. CT and MRI are preferred in detecting small pulmonary metastases and in determining invasion of chest wall muscle, respectively. In predicting whether the adjacent lung is invaded, CT and MRI are not adequate. ${ }^{5}$ PNETs have a very high rate of local recurrence and a propensity to metastasize. The most common sites of dissemination have been reported in bone $(35 \%-50 \%)$, bone marrow (29\%-33\%), lung (18\%-50\%), liver (20\%), lymph node (10\%-12\%) and spleen 

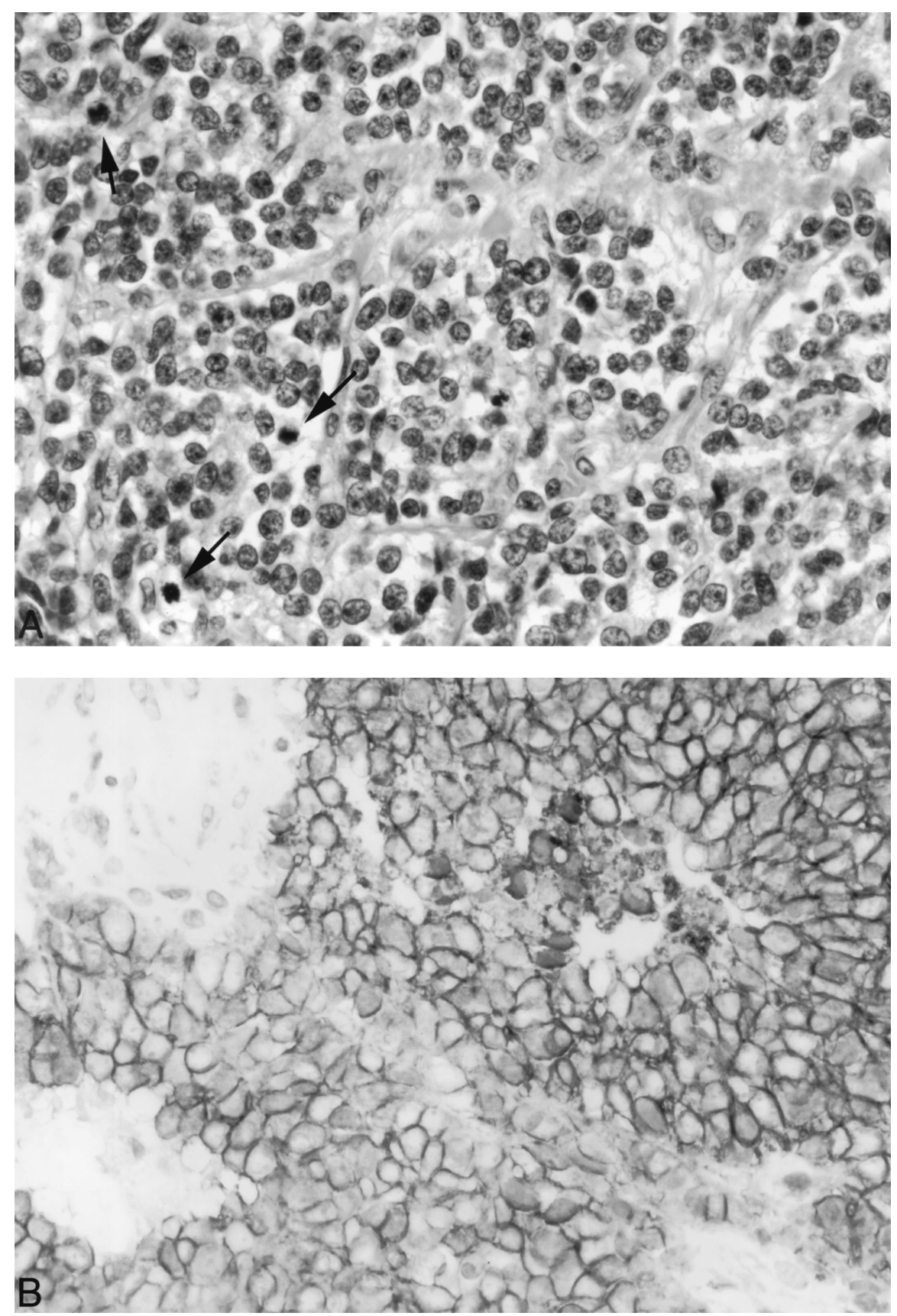

Figure 2. A, Hematoxylin-and-eosin staining of the tumor in patient 2. (Original magnification 250×.) Lobular arranged tumor cells with round-oval nuclei with numerous mitotic ıgures are shown (arrow represents mitotic figures). B, Staining of tumor cells with CD99.

(10\%), and metastases in multiple sites (35\%) have also been seen. ${ }^{6,7}$ Verrill and colleagues ${ }^{8}$ concluded that tumor burden at presentation is the single most important prognostic factor, followed by response to treatment, itself partly determined by tumor size. Age was no prognostic factor in patients with ES or PNET. The PNET is known to be highly chemoresponsive and radioresponsive, as was shown with a high initial complete response of
$94 \%$ in patients treated with vincristine, Adriamycin, and cyclophosphamide chemotherapy plus local radiation therapy. ${ }^{6}$ However, in this study the period of observation was very short.

The best responses were reported with combinations based on anthracyclines (doxorubicin) and high doses of alkylating agents (cyclophosphamide or ifosfamide). ${ }^{7}$ The most frequently used combination protocols include vincristine, actinomycin D, cyclo- 


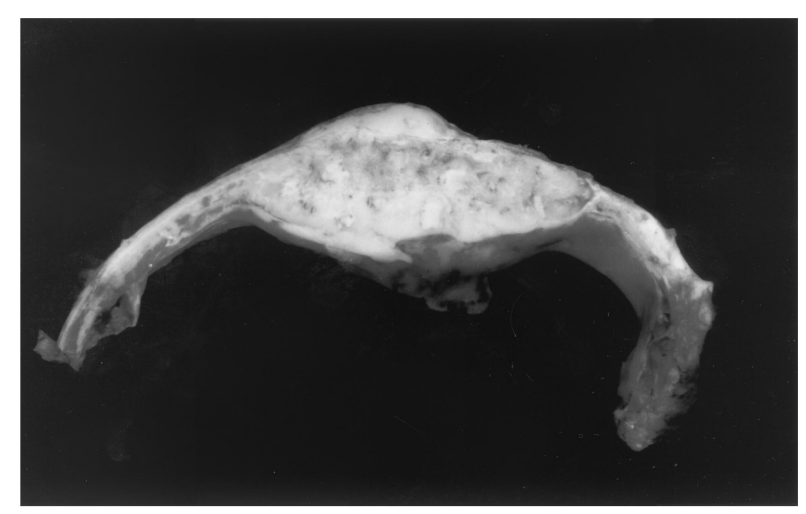

Figure 3. Resection specimen showing a soft-tissue mass of $11 \times$ $7 \times 4.8 \mathrm{~cm}$ with rib involvement.

phosphamide, doxorubicin, and ifosfamide. This is in accordance with the chemotherapy protocols used in our above-described patients.

Etoposide and platinum-based chemotherapy have been used in patients with relapse, although their value has yet to be proven.

A recent phase II trial by Kushner (entitled "Phase II study of the P6 regimen followed by radiotherapy in patients with peripheral neuroectodermal tumors, including Ewing's sarcoma") is investigating the response rate and event-free survival of patients with PNET and ES treated with a chemotherapy regimen with surgical intervention, followed by radiotherapy. Also, the relation between PNET and ES will be further elucidated.
Multimodality treatment, including radical surgical resection, neoadjuvant and adjuvant chemotherapy, and irradiation, is the current treatment of choice. However, the preoperative diagnosis is often very difficult to establish. Survival remains poor, with a 2-year survival of $28 \%$ to $38 \%$ and a 6 -year survival of $14 \%$ to $17 \% .^{3}$

\section{References}

1. Askin FB, Rosai J, Sibley RK, Dehner LP, McAlister WH. Malignant small cell tumor of the thoracopulmonary region in childhood. Cancer. 1979;43:2438-51.

2. Linnoila RI, Tsokos M, Triche TJ, Marangos PJ, Chandra RS. Evidence for neural origin and PAS-positive variants of the malignant small cell tumor of thoracopulmonary region ("Askin tumor"). Am J Surg Pathol. 1986;10:124-33.

3. Contesso G, Llombart-Bosch A, Terrier Ph, et al. Does malignant small round cell tumor of the thoracopulmonary region (Askin tumor) constitute a clinicopathologic entity? Cancer. 1992;69:1012-20.

4. Hicks MJ, Smith JD, Carter AB, Flaitz CM, Barrish JP, Hawkins EP. Recurrent intrapulmonary malignant small cell tumor of the thoracopulmonary region with metastasis to the oral cavity: review of literature and case report. Ultrastruct Pathol. 1995;19:297-303.

5. Winer-Muram HT, Kauffman WM, Gronemeyer SA, Jennings SG. Primitive neuroectodermal tumors of the chest wall (Askin tumors): CT and MR findings. AJR Am J Roentgenol. 1993;161:265-8.

6. Miser JS, Kinsella TJ, Triche TJ, et al. Treatment of peripheral neuroepithelioma in children and young adults. J Clin Oncol. 1987; 5:1752-8.

7. Jürgens H, Bier V, Harms D, et al. Malignant peripheral neuroectodermal tumors. A retrospective analysis of 42 patients. Cancer. 1988; 61:349-57.

8. Verrill MW, Judson IR, Harmer CL, Fisher C, Thomas JM, Wiltshaw E. Ewing's sarcoma and primitive neuroectodermal tumor in adults: are they different from Ewing's sarcoma and primitive neuroectodermal tumor in children? J Clin Oncol. 1997;15:2611-21. 